\title{
Out-of-Hospital Care of Heart Failure Patients During and After COVID-19 Pandemic: Time for Telemedicine?
}

\author{
Alessandro Faragli 1,2,3,4*, Edoardo La Porta ${ }^{5,6,7}$, Carlo Campana ${ }^{8}$, Burkert Pieske ${ }^{1,2,3,4}$, \\ Sebastian Kelle ${ }^{1,2,4}$, Friedrich Koehler ${ }^{9}$ and Alessio Alogna ${ }^{2,3,4}$ \\ ${ }^{1}$ Department of Internal Medicine and Cardiology, Deutsches Herzzentrum Berlin, Berlin, Germany, \\ ${ }^{2}$ Charité-Universitätsmedizin Berlin, Department of Internal Medicine and Cardiology, Campus Virchow-Klinikum, Berlin, \\ Germany, ${ }^{3}$ Berlin Institute of Health (BIH), Berlin, Germany, ${ }^{4}$ DZHK (German Center for Cardiovascular Research), Partner \\ Site Berlin, Berlin, Germany, ${ }^{5}$ Department of Cardionephrology, Clinical Ligurian Institute of High Specialty, Villa Maria Group \\ (GVM) Care and Research, Rapallo, Italy, ${ }^{6}$ Department of Internal Medicine, University of Genoa and IRCSS Azienda \\ Ospedaliera Universitaria San Martino-IST, Genoa, Italy, ${ }^{7}$ Unit of Dialysis, IRCSS Istituto Giannina Gaslini, Ospedale \\ Pediatrico, Genoa, Italy, ${ }^{8}$ Department of Cardiology, Sant'Anna Hospital, ASST-Lariana, Como, Italy, ${ }^{9}$ Center for \\ Cardiovascular Telemedicine, Department of Cardiology and Angiology, Charité-Universitätsmedizin Berlin, Berlin, Germany
}

Keywords: heart failure, COVID-19, home monitoring, body fluids, telemedicine

\section{OPINION LETTER}

The current letter has been driven by the clinical observation of the events that happened in the last months during the coronavirus disease 2019 (COVID-19) pandemic in the European countries, with specific reference to the situation of patients in the North of Italy and in Germany.

\footnotetext{
"Specialists are people who always repeat the same mistake."-Walter Gropius, German architect and founder of the Bauhaus School
}

A 71-year-old male, Caucasian, is affected by chronic heart failure (CHF) New York Heart Association (NYHA) class III and chronic kidney disease stage III. The first diagnosis of CHF has been performed 4 years ago after hospitalization for acute coronary syndrome resulting in a percutaneous coronary intervention with primary stenting. Since then, he has been hospitalized at an average of 1.5 times per year. Two thirds of the patient's hospitalizations were caused by worsening of his chronic body fluid congestion with peripheral edema and impaired renal function, while for one third of the cases, the main cause was volume depletion. This has been manifesting with hypotension and hypokalemia as a result of challenges in managing the correct intake of diuretics and blood pressure-lowering medications.

When admitted to the cardiology ward, such a paradigmatic patient represents a challenge, especially with regard to the body fluid management. This requires a specialized heart failure (HF) team with extensive experience in the field. The clinical approach to such complex patients includes daily physical exam and control of body fluid balance through fluid intake and urine output. On top of this, biomarkers, chest X-ray, lung ultrasound, and, for cardiorenal patients, bioimpedance analysis are performed during the hospitalization to assess the patients' congestion status. Moreover, it requires a fine tuning of medications, diet, and liquid restrictions to achieve a proper balance between body volume and blood pressure.

After recompensation and discharge, the patient is left alone with a single method to monitor himself: a standard weight scale. He weighs himself every day, trying to keep contact with his physician on the phone. He relies on elective appointments in the outpatient clinic, three times a year. 
It is February 2020, and the COVID-19 pandemic takes hold. The patient is not able to get a prompt appointment with his physician. In case of worsening of his clinical condition, he is being told to call the emergency number.

This situation could evolve into three different scenarios:

- No Hospitalization Needed and no SARS-CoV-19 Infection During the Pandemic

The patient independently manages his chronic fluid congestion based on the experience of the past years. He can avoid any contact with COVID $-19^{+}$patients. However, the lack of a proper medical assistance may increase the risk of experiencing a sudden decompensation event. Compared to the time pre-COVID-19 pandemic, his mortality risk may look the same in the short term, but it will probably drastically increase in the medium to long term.

\section{- Clinical Deterioration of the Patient Condition}

The patient constantly deteriorates, gains weight, and his quality of life is strongly affected. He does not get an appointment with his general practitioner, neither in the outpatient clinics. $\mathrm{He}$ manages to survive without an emergency hospitalization, but in a poor condition, for a few weeks or months until the pandemic situation has improved, and he receives medical attention.

\section{- Hospitalization for Acute Decompensation}

The patient gets admitted to the hospital because of an acute exacerbation of his condition. The hospitals are under great pressure because of the pandemic, and the intensive care units have limited capacity. His mortality risk may still be higher than before the pandemic.

\section{- Hospitalization for COVID-19}

The patient is infected with SARS-CoV-19, his condition quickly worsens, and he needs to be quickly hospitalized. The mortality risk in this scenario is possibly the highest.

The last two scenarios are unfortunate and, most importantly, avoidable. However, a drastic change in the management of chronic patients is today, more than ever, of paramount importance. In addition to the direct impact on public health, COVID-19 has been challenging the way of living, the habits, as well as many long-existing cultural and social structures on which societies are based. Maybe for the first time in modern medicine, the major strategy of healthcare policymakers has been to keep patients outside hospitals to avoid the spread of the infection. However, this is not enough, and remote monitoring strategies are necessary for the future of a sustainable healthcare system (1) for many reasons.

We are experiencing since many years a clear mismatch between specialized physicians and patients in need of care $(2,3)$. Aging in western countries has led to an increase in the number of patients with multiple comorbidities (4). Since the very first beginning of medicine and then throughout modern times, healthcare systems have been structured on a face-toface patient-physician interaction. This kind of approach has contributed to a hospital influx of patients during the COVID19 outbreak.

Several European healthcare systems seemed unprepared to fight the pandemic, while many hospitals even contributed to the initial spread of COVID-19. In this scenario, most of the scheduled medical and surgical procedures were rescheduled, while many chronic patients have been temporarily lost at follow-up.

For these reasons, telemedicine has turned from being a "nice to have" approach to an essential requirement (5) for a more efficient system. Chronic HF patients are facing an increased challenge regarding the management of body fluids.

While during a hospitalization, the volume status of the patient is generally addressed by the medical doctors with various methods and solutions such as physical examination, ultrasound, chest X-ray, or blood examinations, at home, the solutions available and specific for the prevention of decompensation events in such patients are relatively limited, as described in Figure 1. We now believe that a natural shift toward home monitoring solutions should be considered and encouraged to manage the patients' body volume during and after the pandemic.

While invasive solutions such as cardioMEMS have already demonstrated to decrease HF patients' hospitalization (6), their utilization has been limited mainly due to invasiveness and related adverse events (7) or lack of penetrance among medical doctors (8). The spectrum of non-invasive telemedicine is, instead, broad, and the recent positive results achieved by Köhler et al. (9) and confirmed by the meta-analysis of Zhu et al. (10) are encouraging.

Audio-video tools able to connect patients and physicians for real-time consultations are widely available and, even if still not extensively adopted, during the pandemic, and the relative lockdown, virtual visits (VVs) represented the first tangible action in favor of a home monitoring of chronic patients, obtaining positive results (11). A recent work published by Salzano et al. (12) was able to show in a cohort of 103 HF patients how a $24 / 7$ audio and video management during the pandemic is able to decrease hospitalizations and mortality compared to a previously observed comparable population in which telemedical support was not present or available. While the feasibility and utility of such solutions for HF patients have been shown to be beneficial even before the pandemic (13), a lot of work is necessary to support a routine utilization. Even if advancements were made in terms of reimbursements, audio/video tools are not yet part of an organized widespread telemonitoring plan in all countries (11). Moreover, VVs require an important engagement by the medical doctors that many times does not match with the time available. Centralized hospitals dedicated only to telemedicine may solve such a problem. The Center for Cardiovascular Telemedicine in Charité Berlin is an example of how a centralized management of telemedicine information is able to act successfully on distant and rural territories (9).

Portable or wearable devices collecting vital parameters while involving the use of Web apps or smartphones are increasingly reliable, and they represent the next generation of solutions available for $\mathrm{CHF}$ patients (14). However, even if most research 


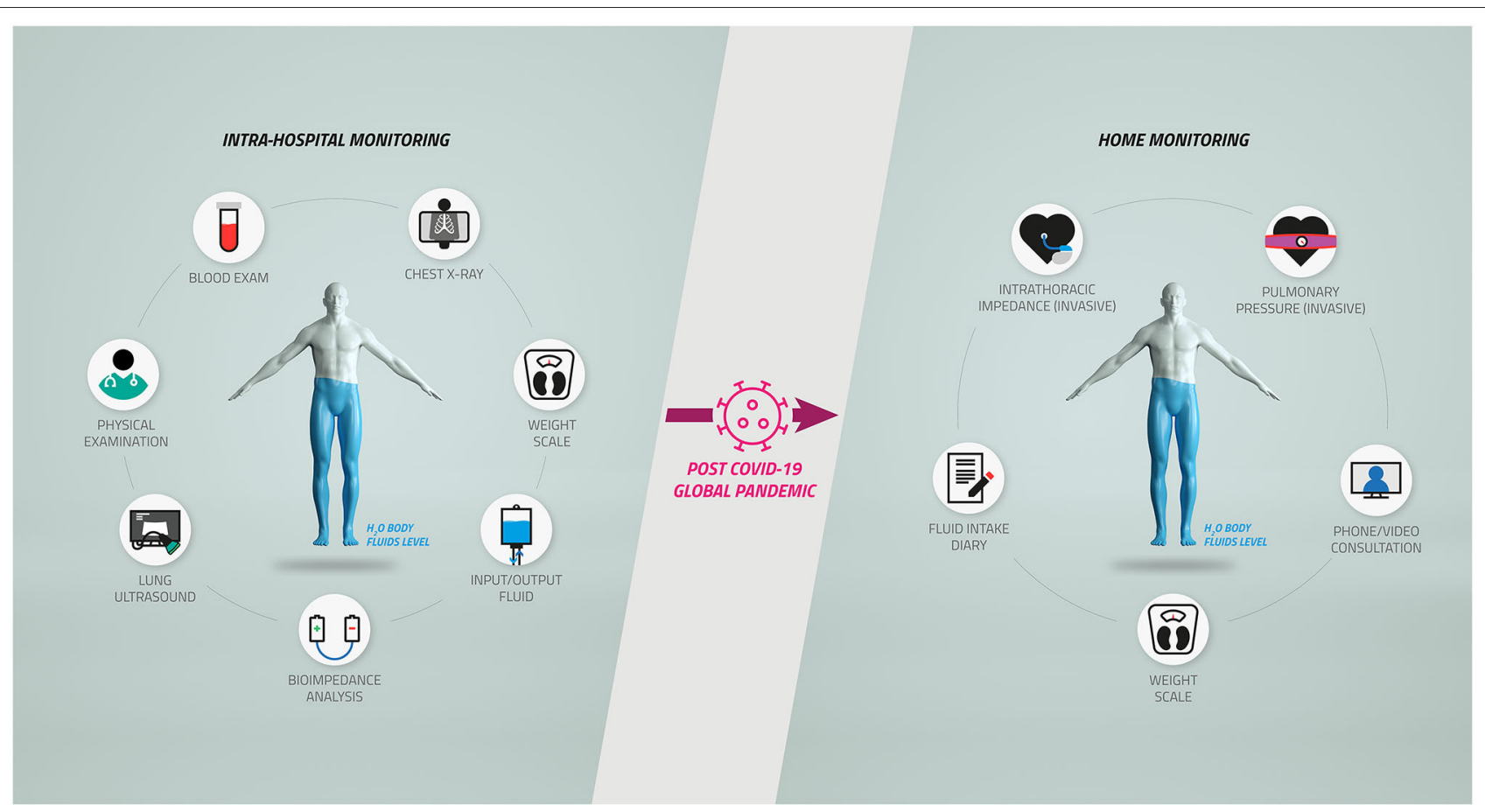

FIGURE 1 | Body fluid management in chronic heart failure and cardiorenal patients after the coronavirus disease 2019 (COVID-19) pandemic. The complex workflow for the diagnosis and monitoring of body fluids of chronic heart failure and cardiorenal patients inside the hospitals (Left) has been restricted by the COVID-19 pandemic, causing a potentially relevant shift toward home monitoring protocols (Right).

has shown the cost-effectiveness of such devices, regulatory authorities have slowed a full penetration of wearables in the medical market until further clinical evidence is available (15).

Another important aspect is technological, since the correct technology should match with the correct clinical indication. For example, for HF patients, a further step should be taken to move beyond the utilization of weight scales, known since many years to be poorly accurate in detecting body fluid congestion and body volume imbalances (14). While remote monitoring through implantable cardioverter defibrillators (ICDs) works really well for the detection of arrhythmias, the same cannot be said for the management of body fluids through intrathoracic impedance mainly due to the high risk of false positives that slowed the initial enthusiasm (16). Even if a lot of research is undergoing in new non-invasive technologies for the assessment of body fluids, their clinical value still needs to be demonstrated (14).

The complex clinical scenario offered by the COVID-19 pandemic should finally be the springboard for telemedicine. Telemedicine still presents challenges, such as the identification of the correct patients' populations in need, a variable that should always be addressed first. This has been nicely demonstrated in a recent randomized, multicenter, open-label telemedicine study by Galinier et al. (17), where patients at higher risk and the ones more socially isolated presented better clinical outcomes than more stable patients, showing how telemedicine may be more useful in such patients. Usability of technologies and increased adherence to the monitoring plan are some of the topics that need to be addressed to finally make telemedicine affordable and efficient for the post-pandemic healthcare system.
On top of that, we believe that optimization of the healthcare organization and automatization of the management processes, meaning data collection, data interpretation, and clinical action toward the patients, need to proceed in a highly structured and fast path to be completely effective. This could be potentially achieved by departments or hospitals dedicated to telemedicine in conjunction with general practitioners. We believe that the introduction of working telemedicine programs needs to enter a novel stage, assigning specific duties and responsibilities to trained personnel. A collaboration between general practitioners and specialized centers is necessary mainly for medically underserved and rural areas. However, the roles need to be precisely defined to avoid confusion.

Both the Heart Failure Society of America and European Society of Cardiology strongly encourage the use of telemedicine for HF management during the COVID-19 outbreak (18). However, a functioning widespread system that allows the reimbursement of home monitoring solutions is still lacking (19). Germany is moving in an innovative direction with the so-called Digital Care Act, entitling all individuals covered by statutory health insurance to reimbursement for certain digital health applications (20). The chance of having a digital solution reimbursed encourages the hospitals to adopt novel telemedical solutions and produce proactively a much faster tangible outcome.

In Italy, during the COVID-19 outbreak, several patients experienced a poor outcome because they did not access to health system (21). Telemedicine owns nowadays the potential of delivering a better healthcare by empowering patients and 
by providing individualized healthcare, especially during a pandemic (22).

Yes, it is time for telemedicine. But, first, let us make telemedicine a matter of routine. Let us learn from our mistakes.

\section{AUTHOR CONTRIBUTIONS}

All authors listed have made a substantial, direct and intellectual contribution to the work, and approved it for publication.

\section{FUNDING}

We acknowledge support from the German Research Foundation (DFG) and the Open Access Publication Fund of CharitéUniversitätsmedizin Berlin. AF and AA received the grant

\section{REFERENCES}

1. Fineberg HV. Shattuck lecture. A successful and sustainable health system-how to get there from here. N Engl J Med. (2012) 366:10207. doi: 10.1056/NEJMsa1114777

2. Scheffler RM, Liu JX, Kinfu Y, Dal Poz MR. Forecasting the global shortage of physicians: an economic- and needs-based approach. Bull World Health Organ. (2008) 86:516-23B. doi: 10.2471/BLT.07.046474

3. Liu JX, Goryakin Y, Maeda A, Bruckner T, Scheffler R. Global health workforce labor market projections for 2030. Hum Resour Health. (2017) 15:11. doi: 10.1186/s12960-017-0187-2

4. Ferrucci L, Fabbri E. Inflammageing: chronic inflammation in ageing, cardiovascular disease, and frailty. Nat Rev Cardiol. (2018) 15:50522. doi: 10.1038/s41569-018-0064-2

5. Duffy S, Lee TH. In-person health care as option B. N Engl J Med. (2018) 378:104-6. doi: 10.1056/NEJMp1710735

6. Abraham WT, Perl L. Implantable hemodynamic monitoring for heart failure patients. J Am Coll Cardiol. (2017) 70:389-98. doi: 10.1016/j.jacc.2017.05.052

7. Vaduganathan M, DeFilippis EM, Fonarow GC, Butler J, Mehra MR. Postmarketing adverse events related to the cardioMEMS HF system. JAMA Cardiol. (2017) 2:1277-9. doi: 10.1001/jamacardio.2017.3791

8. Singh R, Varjabedian L, Kaspar G, Zughaib M. CardioMEMS in a busy cardiology practice: less than optimal implementation of a valuable tool to reduce heart failure readmissions. Cardiol Res Pract. (2018) 2018:49187574918757. doi: 10.1155/2018/4918757

9. Koehler F, Koehler K, Deckwart O, Prescher S, Wegscheider K, Kirwan B-A, et al. Efficacy of telemedical interventional management in patients with heart failure (TIM-HF2): a randomised, controlled, parallel-group, unmasked trial. Lancet. (2018) 392:1047-57. doi: 10.1016/S0140-6736(18)3 1880-4

10. Zhu Y, Gu X, Xu C. Effectiveness of telemedicine systems for adults with heart failure: a meta-analysis of randomized controlled trials. Heart Fail Rev. (2019) 25:231-43. doi: 10.1007/s10741-019-09801-5

11. Gorodeski EZ, Goyal P, Cox ZL, Thibodeau JT, Reay RE, Rasmusson K, et al. Virtual visits for care of patients with heart failure in the Era of COVID19: a statement from the heart failure society of America. J Card Fail. (2020) 26:448-56. doi: 10.1016/j.cardfail.2020.04.008

12. Salzano A, D'Assante R, Stagnaro FM, Valente V, Crisci G, Giardino F, et al. Heart failure management during the COVID-19 outbreak in Italy: a telemedicine experience from a heart failure university tertiary referral centre. Eur J Heart Fail. (2020) 22:1048-50. doi: 10.1002/ejhf.1911

13. Inglis SC, Clark RA, Dierckx R, Prieto-Merino D, Cleland JGF. Structured telephone support or non-invasive telemonitoring for patients with heart failure. (2017) 103:255-7. doi: 10.1136/heartjnl-2015-309191

14. Faragli A, Abawi D, Quinn C, Cvetkovic M, Schlabs T, Tahirovic E, et al. The role of non-invasive devices for the telemonitoring of heart failure patients.
Validation Fund: Track 1 from the Berlin Institute of Health (BIH) on the project cardioBIA: a new algorithm for the prediction and prevention of congestive events in cardiological patients. FK is supported by the grant TELEMED5000 https:// www.telemed5000.de/ founded by the German Federal Ministry of Economics and Energy (BMWi), and the project is associated with the Charité-COVID-19 Research project.

\section{ACKNOWLEDGMENTS}

This short manuscript is dedicated to the loving memory of Giuseppe Faragli, father of AF, heart failure patient, who died during the COVID-19 pandemic. Furthermore, we acknowledge and thank Simone Proietti Timperi for performing the graphical illustration.
Heart Fail Rev. (2020). doi: 10.1007/s10741-020-09963-7. [Epub ahead of print].

15. de la Torre-Díez I, López-Coronado M, Vaca C, Aguado JS, de Castro C. Costutility and cost-effectiveness studies of telemedicine, electronic, and mobile health systems in the literature: a systematic review. Telemed J E Health. (2015) 21:81-5. doi: 10.1089/tmj.2014.0053

16. Böhm M, Drexler H, Oswald H, Rybak K, Bosch R, Butter C, et al. Fluid status telemedicine alerts for heart failure: a randomized controlled trial. Eur Heart J. (2016) 37:3154-63. doi: 10.1093/eurheartj/ehw099

17. Galinier M, Roubille F, Berdague P, Brierre G, Cantie P, Dary P, et al. Telemonitoring versus standard care in heart failure: a randomised multicentre trial. Eur J Heart Fail. (2020) 22:985-94. doi: 10.1002/ejhf.1906

18. Seferovic PM, Ponikowski P, Anker SD, Bauersachs J, Chioncel O, Cleland JGF, et al. Clinical practice update on heart failure 2019: pharmacotherapy, procedures, devices and patient management. An expert consensus meeting report of the heart failure association of the European society of cardiology. Eur J Heart Fail. (2019) 21:1169-86. doi: 10.1002/ejhf.1531

19. Frederix I, Caiani EG, Dendale P, Anker S, Bax J, Böhm A, et al. ESC e cardiology working group position paper: overcoming challenges in digital health implementation in cardiovascular medicine. Eur J Prev Cardiol. (2019) 26:1166-77. doi: 10.1177/2047487319832394

20. Gerke S, Stern AD, Minssen T. Germany's digital health reforms in the COVID-19 era: lessons and opportunities for other countries. NPJ Dig Med. (2020) 3:94. doi: 10.1038/s41746-020-0306-7

21. De Filippo O, D'Ascenzo F, Angelini F, Bocchino PP, Conrotto F, Saglietto A, et al. Reduced rate of hospital admissions for ACS during Covid-19 outbreak in Northern Italy. (2020) 383:88-9. doi: 10.1056/NEJMc2009166

22. Cleland JGF, Clark RA, Pellicori P, Inglis SC. Caring for people with heart failure and many other medical problems through and beyond the COVID-19 pandemic: the advantages of universal access to home telemonitoring. Eur J Heart Fail. (2020) 22:995-8. doi: 10.1002/ejhf.1864

Conflict of Interest: AF and ELP are shareholders of the company BOCAhealthcare GmbH.

The remaining authors declare that the research was conducted in the absence of any commercial or financial relationships that could be construed as a potential conflict of interest.

Copyright (C) 2020 Faragli, La Porta, Campana, Pieske, Kelle, Koehler and Alogna. This is an open-access article distributed under the terms of the Creative Commons Attribution License (CC BY). The use, distribution or reproduction in other forums is permitted, provided the original author(s) and the copyright owner(s) are credited and that the original publication in this journal is cited, in accordance with accepted academic practice. No use, distribution or reproduction is permitted which does not comply with these terms. 